\title{
Construction of a 3D Model of Epitympanic Folds and Space
}

\author{
Murugan Kutty Gopalan and Unnikrishnan K. Menon
}

This article reports a technique for making a 3 dimensional (3D) model of the epitympanic folds and spaces in the middle ear. It is intended as a teaching model for both undergraduate and post-graduate medical students.

\section{OPEN ACCESS}

\section{Introduction}

Middle ear anatomy is complex and challenging for anatomists and otologists. The knowledge of epitympanic folds and spaces has particular relevance when considering the etiopathogenesis and spread of cholesteatoma (a destructive and expanding growth consisting of keratinizing squamous epithelium) in the middle ear cleft and related surgery.

The topic is usually covered in the embryology of the middle ear. However, it is not an easily accessible or demonstrable area. The traditional method of cadaveric temporal bone dissection is not very useful in understanding this region. Standard textbooks and literature provide minimal description and few diagrams from which a precise $3 \mathrm{D}$ view does not emerge. Post-graduate anatomy and ENT students have to use their imagination to fully comprehend these small areas.

In such a setting, our 3D model of the middle ear box, showing the ossicles and the related epitympanic folds and spaces, would be of immense help. Here we describe the methodology of making such a model which can serve as an addition to the anatomy and ENT section teaching aids. We are confident that this will arouse interest in the budding anatomists to study this area in detail and greatly help the ENT post-graduates to decipher a complicated area of anatomy.

Endoscopic ear surgery is an upcoming field in otology, where the focus of attention is mainly the epitympanum (attic) and mesotympanum. The ENT surgeon interested in this field would have to undergo specific training in the exploration of the former area. It is hoped that the present model will also be a valuable aid in this aspect.

\section{Objective}

To construct an enlarged three-dimensional model of the epitympanum, demonstrating the folds attached to the ear ossicles and walls of tympanic cavity, and the various spaces. Our intention is that this can be used as a teaching aid.

\section{Materials}

Available literature and diagrams from standard texts, endoscopic pictures from relevant publications, rectangular jar of transparent perspex /acrylic, thermofoam / thermocol pieces, colored transparent thick polythene / glass paper sheets, scissors, sharp razors, iron wires, pins, adhesive gum, etc.

\section{Methods}

We made a detailed study of the available pictures and descriptions (Figures 1 to 3 ), followed by step-by-step construction of each fold and space in a box.

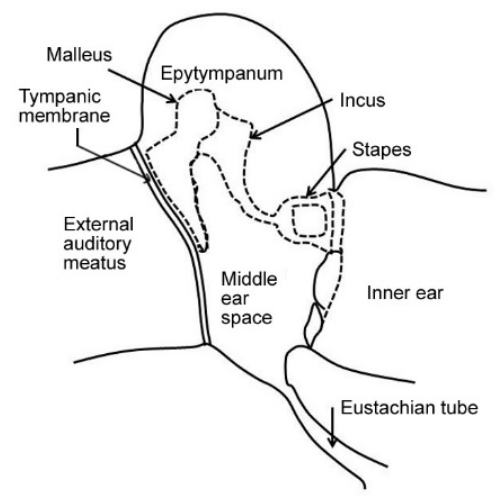

Figure 1a. General topography of middle ear boundaries \& contents (line diagram drawn by the authors.

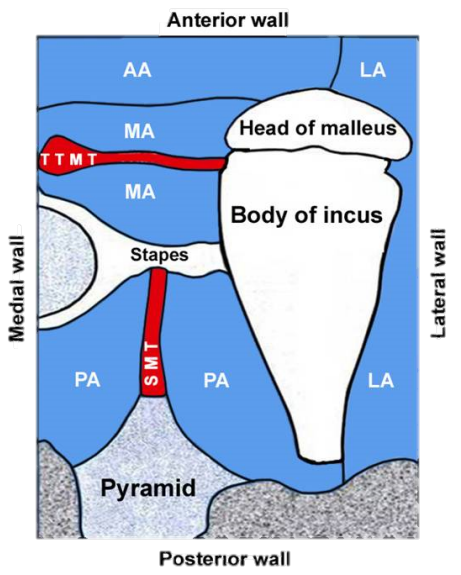

Figure 1b. Epitympanic spaces, superior view - AA, MA, PA, LA, TTMT, SMT (fold and ligaments removed, indicated by blue spaces). Adapted from Wright and Valentine 2008. 


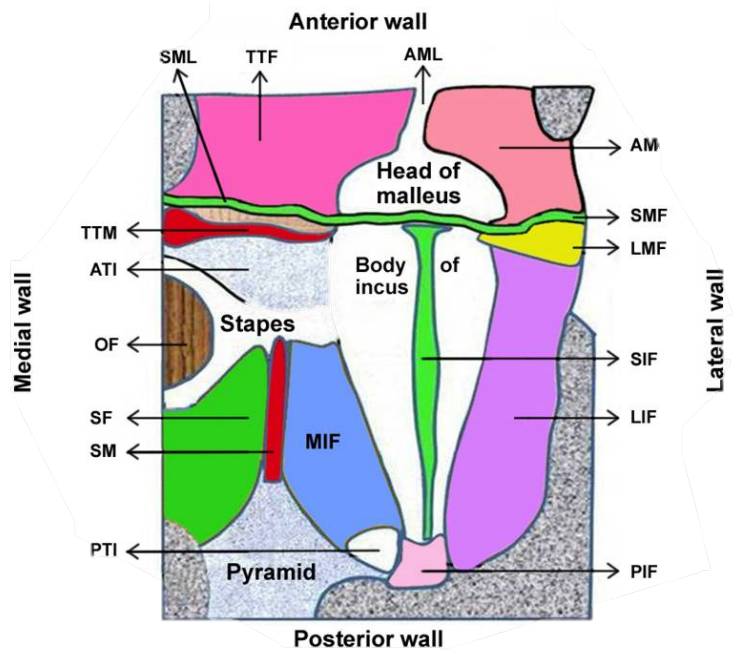

Figure 2. Mucosal folds and ligaments attached to malleus, incus and stapes - AML, SMF, LMF, PMF, TTF, SIF, LIF, MIF, PIF, OF, SF, AML, SML, TTM, ATI, SM, PTI. Adapted from Wright and Valentine 2008.

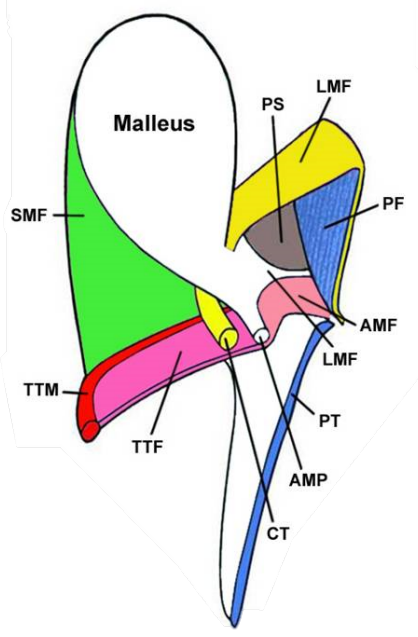

Figure 3. Anterior wall of middle ear removed - view from front to show Prussak's space. LMF, PF, AMF, AMP, CT, SMF, LMP, TTM, TTF, PT, PS. Adapted from Wright and Valentine 2008.

\section{Making of the Model}

Our starting point was a rectangular box. This was in keeping with the standard teaching description of the middle ear as a box-like cavity within 6 walls. We used an acrylic museum jar (made of acrylic sheets for keeping formalin preserved human tissues in the anatomy museum) with the lid removed. A semicircular fold was cut from a transparent glass paper sheet (tympanic membrane) and its peripheral margin was attached to a curved iron wire (tympanic annulus). This was fixed to one side of the box from inside (considered as the lateral wall of the middle ear) with its convexity (umbo) touching the base and facing the interior of the box. The ear ossicles (malleus, incus and stapes) were carved in thermocol and fixed as shown in Figure 4.

Then the various folds cut in glass paper sheets of different colors, muscles made of cloth and pyramid carved in thermocol, were fixed into the box in the anatomical positions using the illustrations given in Figures 2 and 3.

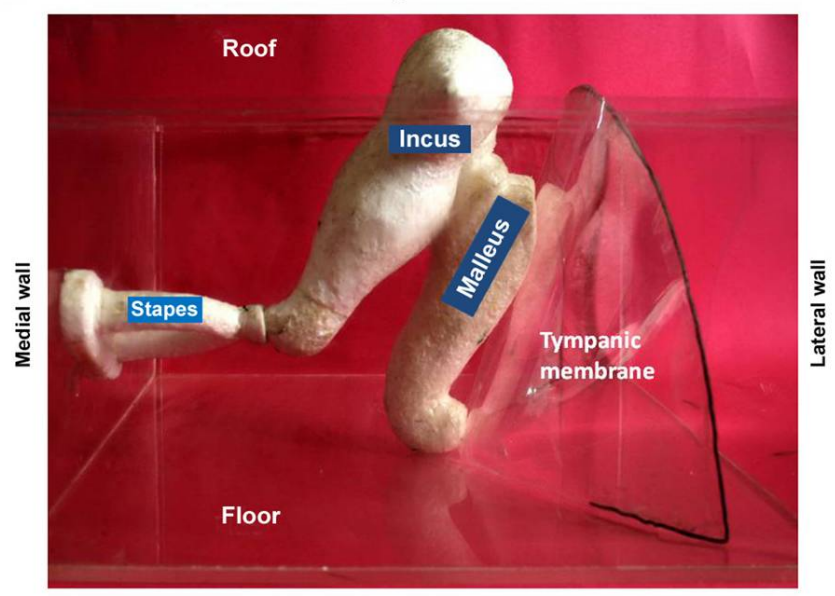

Figure 4. The model middle ear box with the ossicles and tympanic membrane fixed.

Posterior malleolar fold (PMF) (dark violet) fixed into a groove made in the handle of the malleus, was stretched to the peripheral margin of the tympanic annulus.

Interosseous fold (IOF) (orange) was placed between the long processes of malleus and incus (Figure 5).

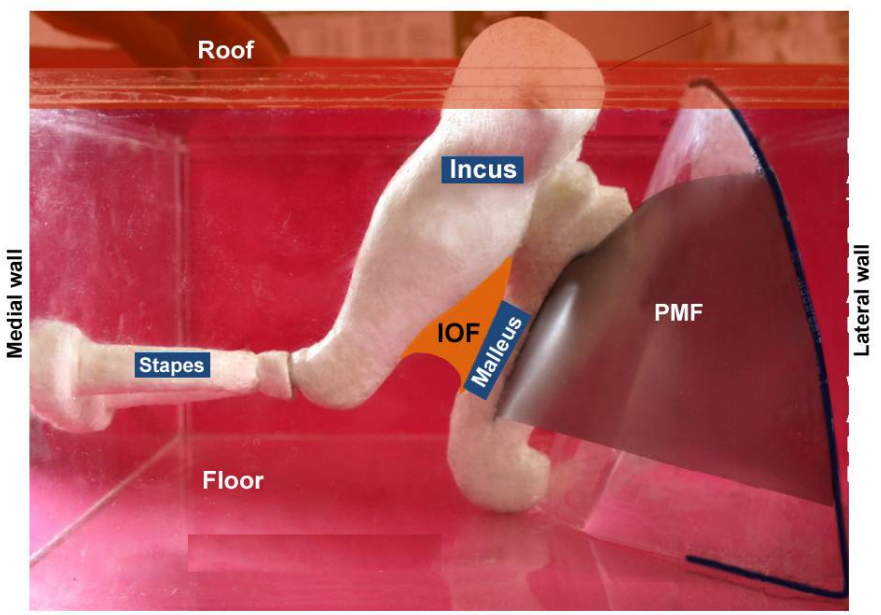

Figure 5. Model (posterior view) - PMF, IOF.

Lateral malleolar fold (LMF) (yellow) stretching from the neck of the malleus up to the scutum (outer attic wall) forming the superior border of Prussak's space (Figure 6).

Lateral incudal fold (LIF) (violet) was fixed, stretching between the long process of the body of the incus and the lateral attic wall. Pyramid was fixed to the posterior wall. The stapedius muscle tendon (SMT) had one end glued in the summit of the pyramid and the other end to the neck of stapes. Medial incudal fold (MIF) (blue) was fitted between the long process of body of the incus and the stapedius muscle and pyramid. Next, the stapedial fold (SF) (dark green) was stretched between the posterior crura of the stapes, the posterior tympanic wall, the 


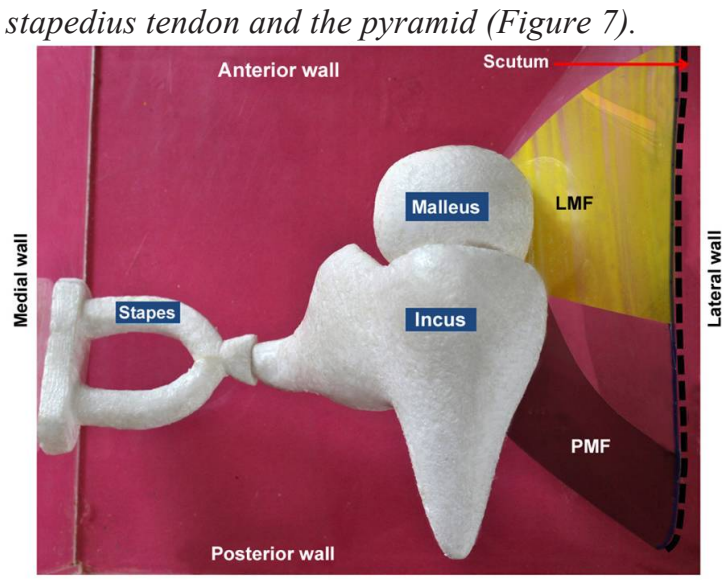

Figure 6. Model (superior view) - PMF, LMF.

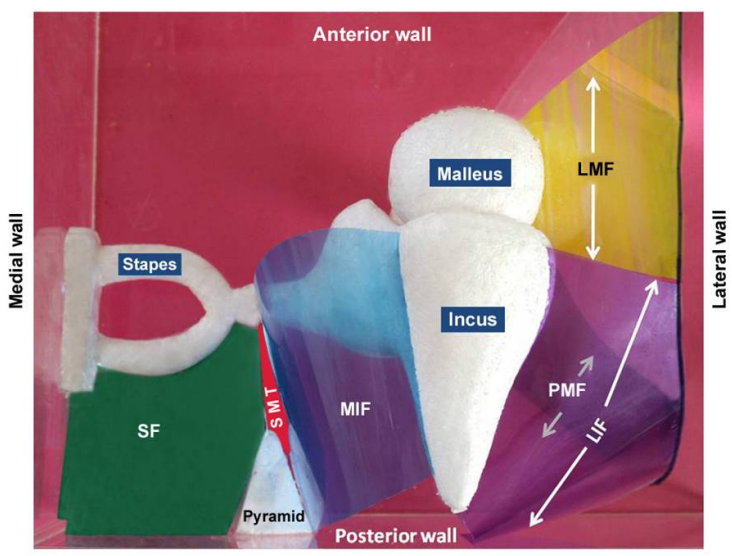

Figure 7. Model (superior view) - LIF, MIF, SF, TM, PMF, SMT.

Anterior malleolar fold (AMF) (maroon) was fixed to extend between the anterior surface of the malleus head and the anterior bony wall of the attic. The tensor tympani muscle tendon (TTMT) was fixed between the handle of the malleus and the medial wall. The tensor tympani fold (TTF) (pink), was attached between the tensor tympani muscle tendon (TTMT) and the anterior plate of the attic wall. The obturator fold (OF) (wood design) was fitted between the two crura of the stapes (Figure 8).

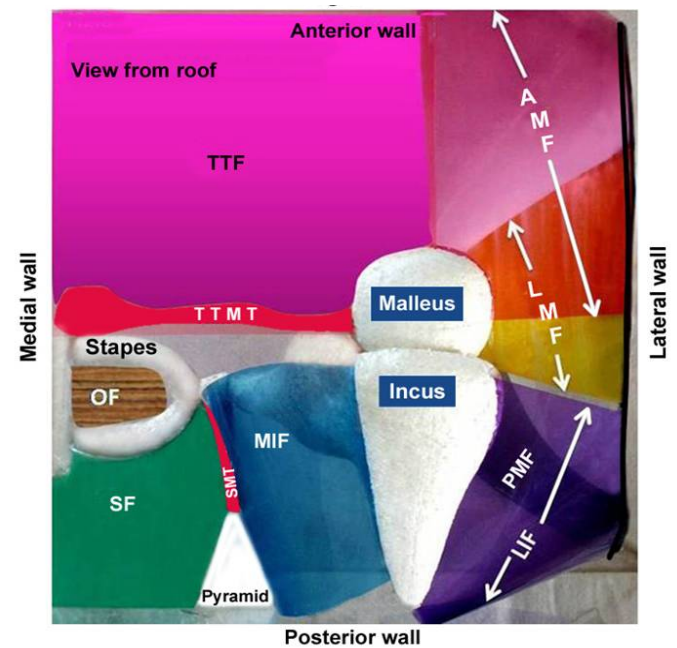

Figure 8. Model (superior view) - AMF, TTMT, TTF, OF, SMT, $M I F, L M F, S F, P M F$.

The superior malleolar fold (SMF) (green) was placed across the cavity between the superior surface of the malleus head and the superior attic wall. The superior incudal fold (SIF) (parrot green) was fixed, extending onto the superior aspect of body of the incus and the superior attic wall. The posterior incudal fold $(P I F)$ (pink) was inserted extending from posterior end of body of the incus to the posterior wall of the attic (Figure 9).

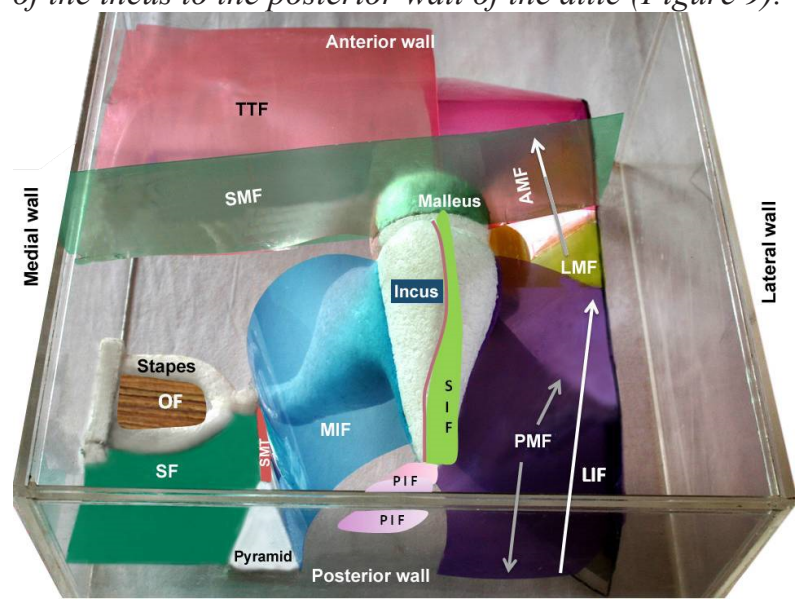

Figure 9. Model (superior view) - SIF, PIF

The lid of the jar (acrylic box) was replaced and screwed into place to produce the final assembly of the model, showing all middle ear structures in position (Figure 10). 


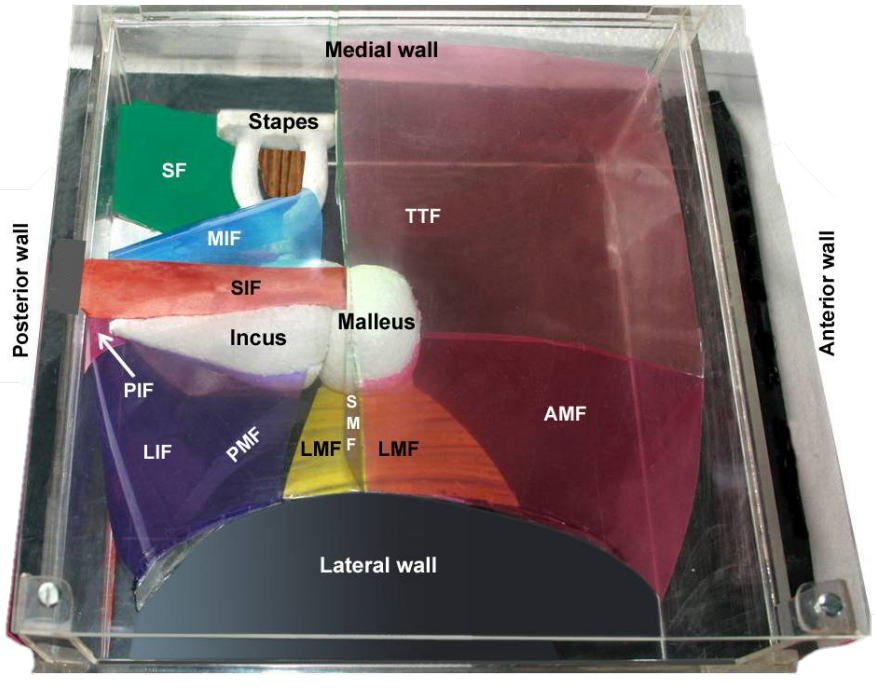

Figure 10. Model (superior view; final assembly) - TTF, MIF, $S I F, P I F, L I F, P M F, S M F, L M F, A M F$.

Viewed from the lateral wall the tympanic membrane was divisible into pars flaccida (PF) and pars tensa (PT). Prussak's space was seen bound by the neck of the malleus medially, pars flaccid (PF) laterally, lateral malleolar fold (LMF) superiorly, anterior malleolar fold (AMF), as well anterior malleolar ligament (AML), anteriorly and lateral malleolar process inferiorly (Figure 11).

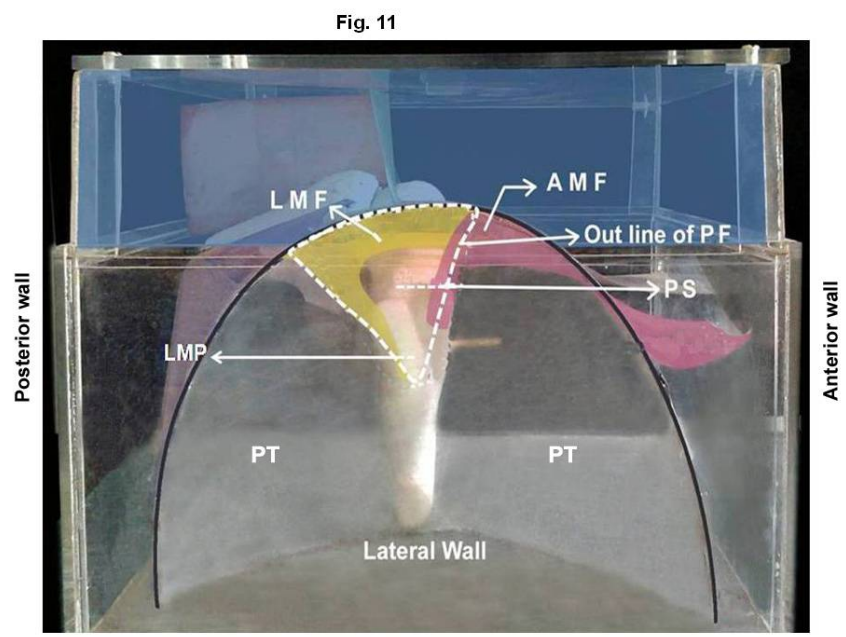

Figure 11. Model showing the lateral wall and Prussak's space$P F, P T, P S, L M F, A M F$ LM.P

\section{Results}

A 3D model of the middle ear cavity with the epitympanum (showing the folds, ligaments and spaces) was made successfully (Figure 9). Ventilation routes to both anterior and posterior epitympanic spaces (and particularly to Prussak's space were well demonstrated and easily visualized (Figures 10 and 11).

\section{Discussion}

The middle ear cavity is divided into the epitympanum (attic), mesotympanum and hypotympanum (Figure 1). The mesotypanum is generally well-described in most standard textbooks. It is also well-demonstrated and studied during the standard temporal bone dissections. However, this is not the case of the epitympanum. This area is much smaller in size and is sub-divided into many spaces, created by several discrete membranous folds stretched between the ossicles and the bony walls of the epitympanum (Figure 1(a) AA - anterior attic, MA medial attic, PA - posterior attic, LA - lateral attic).

Epitympanum is separated from the mesotympanum by the tympanic diaphragm which is formed of mucous folds \& spaces (Figure 2). Two tiny openings, the anterior tympanic isthmus (ATI) and posterior tympanic isthmus (PTI), (Figure 2) with a patent eustachian tube permit ventilation of the middle ear, from mesotympanum to attic, as well to mastoid antrum and vice versa, which is essential for normal hearing (Tos 1995).

Bruce Proctor described the development of the middle ear spaces (Proctor 1964); the concept of epitympanic folds and spaces was a result of his publication. However, this description and figures have proved difficult to understand and teach.

The clinical relevance of these folds and spaces lie in their embryology, and their role in the pattern of spread of cholesteatoma. The latter is one of the common ear pathologies which involve the spread of epithelium into the middle ear cleft, either because of a severely retracted tympanic membrane or through a perforation within it. This spreads into the epitympanum, and thence into the mastoid air cells. The folds initially act as barriers against this, while the spaces provide the passageway into the anterior protympanum, posterior antrum, and mastoid air cells. Amongst these spaces, Prussak's space is considered the most significant, as it allows the disease to spread from the epitympanum to the mesotympanum, anterior to the handle of malleus. The ear surgeon has to be aware of this pathway of spread.

Of recent interest, the endoscopic approach to ear surgery also warrants a detailed knowledge of this area (Pothier 2013). Radiologists and neurosurgeons, with specific interest in the temporal bone, would also benefit from precise knowledge of this area.

The available literature on this part of the ear anatomy is not very descriptive. Textbooks provide short descriptions and $2 \mathrm{D}$ pictures (Tos 1995; Wright 2008). However, these do not give a precise, $3 \mathrm{D}$ view of the epitympanic spaces.

Given this background, solid methods with which to study this area of the middle ear would be of great use. It has been our endeavor to contribute something in this regard. The result is a magnified, three-dimensional model, which is easily manufacturable and portable.

\section{Conclusion}

The present model would be valuable as a teaching aid for undergraduate and postgraduate students, Neuro-ENT surgeons and radiologists in order to have a $3 \mathrm{D}$ orientation of the structures in epitympanum. It would be helpful in the following instances: 
- For the ENT surgeon interested in endoscopic micro ear surgery

- For radiologists in the detailed reporting of a high resolution computerized

- tomography of the temporal bone

- For neurosurgeons dealing with temporal bone lesions

- Understanding the concept of selective epitympanic dysventilation

\section{Acknowledgements}

We would like to acknowledge the invaluable technical support provided by Mr. M. G. Ramachandran (artist-modeler) and Mr. M. M. Varghese (photographer) in the preparation of the model and photographs for the article.

\section{List of Abbreviations}

\author{
AA - anterior attic \\ AMF - anterior malleolar fold \\ AML - anterior malleolar ligament \\ AMP - anterior malleolar process \\ ATI - anterior tympanic isthmus \\ CP - cochleariform process \\ CT - chorda tympani \\ ET - epitympanum \\ FN -facial nerve \\ I - incus \\ IOF - inter-osseous fold \\ LC - lateral semicircular canal \\ LA - lateral attic \\ LIF - lateral incudal fold \\ LMF - lateral malleolar fold \\ LMP - lateral aspect of malleolar process \\ $\mathrm{M}$ - malleus \\ MA - medial attic \\ MIF - medial incudal fold \\ OF - obturator fold \\ PA - posterior attic \\ PE - pyramidal eminence \\ PES - posterior epitympanic space \\ PF - pars flaccida \\ PIF - posterior incudal fold \\ PMF - posterior malleolar fold \\ PS - Prussak's space \\ PT - pars tensa \\ PTI - posterior tympanic isthmus \\ $\mathrm{S}$ - stapes \\ SF - stapedial fold \\ SIF - superior incudal fold \\ SM - stapedius muscle \\ SMF - superior malleolar fold \\ SML - superior malleolar ligament \\ SMT - stapedius muscle tendon \\ TTF - tensor tympani fold \\ TTM - tensor tympani muscle \\ TTMT - tensor tympani muscle tendon
}

\section{References}

Pothier D.D. 2013. Introducing Endoscopic Ear Surgery into Practice. Otolaryngology Clinics of North America, 46(2): 245-55.

Proctor B. 1964. The development of the middle ear spaces and their surgical significance. The Journal of Laryngology and Otology, 78(7): 631-648.

Tos, Mirko. 1995. Manual of Middle Ear Surgery, Vol. 2. New York: Thieme Medical Publishers Inc.

Wright, Tony and Valentine, Peter. 2008. The anatomy and embryology of the external and middle ear. In ScottBrown's Otorhinolaryngology and Head and Neck Surgery, 7th ed. Vol 3. London: Hodder Arnold.

\section{Authors}

Both authors belong to the teaching Faculty of Amrita Institute of Medical Sciences and Research Centre, Amrita University, which is a teaching institute attached to a multi-specialty tertiary referral hospital, in the southern state of Kerala, India.

Dr. Murugan Kutty Gopalan BSc, MBBS, DMA (USA) Dr. Murugan Kutty Gopalan B.Sc, MBBS, DMA (USA) is the Head of the Departments of Medical Illustrations, Center for Digital Health and Amrita Clinical Skills Simulation center in the School of Medicine, Amrita Institute of Medical Sciences \& Research Center, Amrita University in Kerala, India. He has been teaching human anatomy for undergraduate and postgraduate students of medicine, Dentistry and Nursing for the past 35 years. He has broad expertise in medical sculpture/artwork and has constructed a number of 3D illustrative models (physical) using various media for educational purposes in the fields of human anatomy, microbiology, pathology, medical and surgical specialties. He has recently developed more than 500 instant, innovative, cost effective 3D anatomical teaching aids, and conducted workshops for anatomy faculty members and modelers, at six nodal centers in India to train them to make these teaching aids. $\mathrm{He}$ is also involved in giving training in various clinical skills using low and high fidelity mannequins. He is intensely working on introducing new generation medical haptics, robotic surgery, cardiac-neuro-ortho interventional simulaids for the super-specialty. Dr.Gopalan has won several regional, national and international awards for his innovative works. He can be contacted at murugankuttygopalan@gmail.com. 
Dr. Unnikrishnan K. Menon, MS [ENT], DNB [ENT], is Clinical Associate Professor in the Department of Ear, Nose and Throat, School of Medicine, Amrita Institute of Medical Sciences and Research Centre, Amrita University, Kerala, India, a teaching Institute attached to a multi-specialty tertiary referral hospital. In addition to routine surgical work, his chief area of interest is the teaching of basic sciences to postgraduate students. He has worked extensively with the first author in making several ENT-related models to enhance understanding of anatomy and physiology. He can be contacted at unnikrishnanmenon8@gmail.com

\section{Licensing}

The authors have chosen to license their content under a Creative Commons Attribution, NonCommercial, NoDerivatives License 4.0 International License.

\section{(C) $(1) \Theta$}

Construction of a 3D Model of Epitympanic Folds and Space by Murugan Kutty Gopalan and Unnikrishnan K. Menon

Journal of Biocommunication, 40 (1), 2016

http://journals.uic.edu/ojs/index.php/jbc/article/view/6628

DOI: $\underline{\text { http://dx.doi.org/10.5210/jbc.v40i1.6628 }}$ 